\title{
Inclusion of pterostilbene in natural cyclodextrins: complex preparation and solid-state characterization
}

\author{
Laura Catenacci ${ }^{*}$, Milena Sorrenti ${ }^{1}$, Maria Cristina Bonferoni ${ }^{1}$, Alexios I. Vicatos ${ }^{2}$ and Mino R. \\ Caira $^{2}$ \\ 1 Department of Drug Sciences, University of Pavia, Viale Taramelli 12, 27100 Pavia, Italy; \\ laura.catenacci@unipv.it; milena.sorrenti@unipv.it; mariacristina.bonferoni@unipv.it. \\ 2 Department of Chemistry, University of Cape Town, Rondebosch 7701, South Africa; \\ Mino.Caira@uct.ac.za; VCTALE001@myuct.ac.za. \\ * Correspondence: laura.catenacci@unipv.it; Tel.: +39-382-987054 \\ Received: 23 November 2020; Accepted: date; Published: date
}

\begin{abstract}
Pterostilbene (3,5-dimethoxy-4'-hydroxystilbene, PTB) is a natural dietary polyphenol, occurring primarily in blueberries and Pterocarpus marsupium heartwood. In recent years, this compound has attracted increasing interest owing to its antioxidant, anti-inflammatory and anticarcinogenic properties and its capacity to reduce and regulate cholesterol and sugar blood levels. The low aqueous solubility of pterostilbene is one factor that limits its utility, and among the different methods available for its improvement is its complexation with cyclodextrins (CDs), the subject of this study. CDs are natural macrocyclic oligomers composed of $\alpha$-D-glucose units linked by $\alpha-1,4$ glycosidic bonds to form torus-shaped molecules. This conformation is responsible for inclusion complex formation with organic molecules, the latter generally being included within the hydrophobic $\mathrm{CD}$ cavity. In this study, various methods used to prepare inclusion complexes between PTB and three natural cyclodextrins $(\alpha-C D, \beta-C D$ and $\gamma-C D)$ are described. In addition, the isolation of single crystals of complexes of PTB with $\beta-\mathrm{CD}$ and $\gamma-\mathrm{CD}$ was pursued to determine the nature of their host-guest interactions using $X$-ray diffraction.
\end{abstract}

Keywords: Pterostilbene; Cyclodextrin; Complexation; Thermal Analysis; X-ray Diffraction

\section{Introduction}

Pterostilbene (3,5-dimethoxy-4'-hydroxystilbene, PTB) is a natural dietary polyphenol belonging to the stilbene class, occurring primarily in blueberries and Pterocarpus marsupium heartwood [1]. PTB has attracted increasing interest owing to its several therapeutic effects, namely anticancer, hypolipidemic and anti-diabetes activities, in addition to its antioxidant and antiinflammatory activities [2]. PTB is a methoxylated derivative of resveratrol, characterized by low aqueous solubility and high lipophilicity (Class II drug in the Biopharmaceutics Classification System, BCS) and its low solubility is one of the principal factors that limits its utility.

Among the different methods available for the solubility improvement, complexation with cyclodextrins (CDs) is a promising approach and is investigated in this study [3].

CDs are natural macrocyclic oligomers composed of $\alpha$-D-glucose units linked by $\alpha-1,4$ glycosidic bonds to form torus-shaped molecules. This conformation is responsible for inclusion complex formation with organic molecules, the latter generally being included within the hydrophobic CD cavity. In addition to enhancing the solubility of guest molecules, CD complexation can be used to improve other physicochemical properties such as chemical stability, to prevent gastrointestinal irritation, to reduce or eliminate unpleasant odours and flavours, and to prevent drug-drug or drugexcipient interactions [4].

The 1st International Electronic Conference on Pharmaceutics, 1 - 15 December 2020 
In the attempt to obtain CD.PTB complexes, various methods were investigated, including solution-phase techniques, such as co-precipitation and evaporation with microwave irradiation and rotavapor, the kneading method, which yields a paste, and mechanical grinding methods.

The characterization of all the solid phases isolated was performed using differential scanning calorimetry (DSC), thermogravimetric analysis (TGA), X-ray diffraction (XRD), Fourier transform infrared (FT-IR) and ${ }^{1} \mathrm{H}$ NMR spectroscopy.

\section{Experiments}

\subsection{Materials}

The PTB sample used was purchased from Mega Resveratrol Candlewood Stars Inc. (Danbury CT, USA). The native CDs were supplied by Wacker Chemie Italia Srl (Milan, Italy) and Cyclolab (Budapest, Hungary). All other materials and solvents used were of analytical reagent grade.

\section{Sample preparation}

Physical mixtures (PMs) were prepared by manual trituration of equimolar amounts of PTB with each of the natural CDs using a mortar and pestle. Each PM was then wetted with ethanol and dried to constant mass at $70{ }^{\circ} \mathrm{C}$ in an oven to obtain the kneaded product $(\mathrm{KN})$.

\subsection{Methods}

\section{Differential Scanning Calorimetry (DSC)}

DSC analyses were performed with a Mettler STARe system (Mettler Toledo, Milan, Italy) equipped with a DSC821 $\mathrm{e}$ Module and an Intracooler device for sub-ambient temperature analysis (Julabo FT 900) on $2-4 \mathrm{mg}$ (Mettler M3 Microbalance) samples in sealed aluminium pans with pierced lid (heating rate $\beta=10 \mathrm{~K} \mathrm{~min}^{-1}$, nitrogen air atmosphere, flux $50 \mathrm{ml} \mathrm{min}^{-1}$ ), range temperature: $30-350{ }^{\circ} \mathrm{C}$ ). The instrument was previously calibrated with Indium as standard reference and the measurements were carried out at least in triplicate.

Simultaneous Thermogravimetric Analysis (TGA)

Mass losses were recorded with a Mettler STARe system (Mettler Toledo, Milan, Italy) TGA instrument with simultaneous DSC (TGA/DSC1) on $4-6 \mathrm{mg}$ samples in alumina crucibles with lid (heating rate $\beta=10 \mathrm{~K} \mathrm{~min}^{-1}$, nitrogen air atmosphere, flux $50 \mathrm{ml} \mathrm{min}^{-1}$ ), temperature range: 30-350 $\left.{ }^{\circ} \mathrm{C}\right)$. The instrument was previously calibrated with Indium as standard reference and measurements were carried out at least in triplicate.

\section{Fourier Transform Infrared Spectroscopy (FT-IR)}

A spectrophotometer Perkin Elmer Spectrum One (Perkin Elmer, Monza, Italy) with a single reflection ATR accessory (Pike MIRacle ${ }^{\mathrm{TM}}$ Technologies, Madison, Wisconsin, USA) was used for Fourier transform infrared (FT-IR) mid-IR spectroscopy $\left(650-4000 \mathrm{~cm}^{-1}\right)$ of the samples. Each sample was placed on an ATR crystal of ZnSe and pressed onto the crystal. The spectra were collected in transmittance mode with 64 scans and a resolution of $4 \mathrm{~cm}^{-1}$.

\section{${ }^{1 .}$ H NMR spectroscopy}

${ }^{1} \mathrm{H}$ NMR spectroscopy was used to determine the host-guest ratio for the CDיPTB complex. Separate solutions of the host and the guest were prepared in deuterated dimethyl sulfoxide (DMSO$\mathrm{d}_{6}$ ). Single crystals of the inclusion complex were likewise dissolved in DMSO-d6. The proton NMR spectra of all three solutions were recorded on a Varian-Gemini 300 spectrometer (Varian, Inc., Palo Alto, CA, USA) at $298 \mathrm{~K}$. 


\section{X-ray diffraction}

Powder X-ray diffraction (PXRD) patterns were collected on a Bruker D5005 powder diffractometer (Siemens, Germany) equipped with a $\theta-\theta$ vertical goniometer and a Position Sensitive Detector (PSD, Braun). $\mathrm{CuK}_{\alpha}$ radiation $(\lambda=1.5418 \AA$ ) $)$ was employed with generator settings $40 \mathrm{kV}$ and $30 \mathrm{~mA}$.

Patterns were recorded in the step-scan mode (step: 0.015, counting time: $0.5 \mathrm{~s}$ ) in the angular range $5^{\circ}<2 \theta<30^{\circ}$ at room temperature.

For single crystal X-ray diffraction of the CD inclusion complexes of PTB a specimen of high crystalline quality was mounted on a nylon cryoloop with Paratone oil (Exxon Chemical Co., TX, USA) and placed on a Bruker Apex II diffractometer for intensity data-collection. Data-reduction and scaling were performed using program SAINT. The structures were solved and refined by full-matrix least squares using programs in the SHELX suite.

\section{Results}

\subsection{Thermal and FT-IR characterization of the commercial product}

Commercial PTB is a white crystalline powder, with a thermal profile typical of an anhydrous sample (Figure 1). The DSC curve (a) shows a single endothermic effect at $96 \pm 0.3{ }^{\circ} \mathrm{C}($ Tonset $=95.5 \pm$ $0.2{ }^{\circ} \mathrm{C} ; \Delta \mathrm{H}_{\mathrm{m}}=97 \pm 2 \mathrm{Jg}^{-1}$ ) related to the melting of the sample. The subsequent endothermic effect, at approximately $220^{\circ} \mathrm{C}$ is attributable to the melt decomposition.

The TGA analysis (b) of the sample confirms the anhydrous nature of PTB, displaying only one mass loss recorded just after the melting temperature range in DSC due to thermal decomposition.

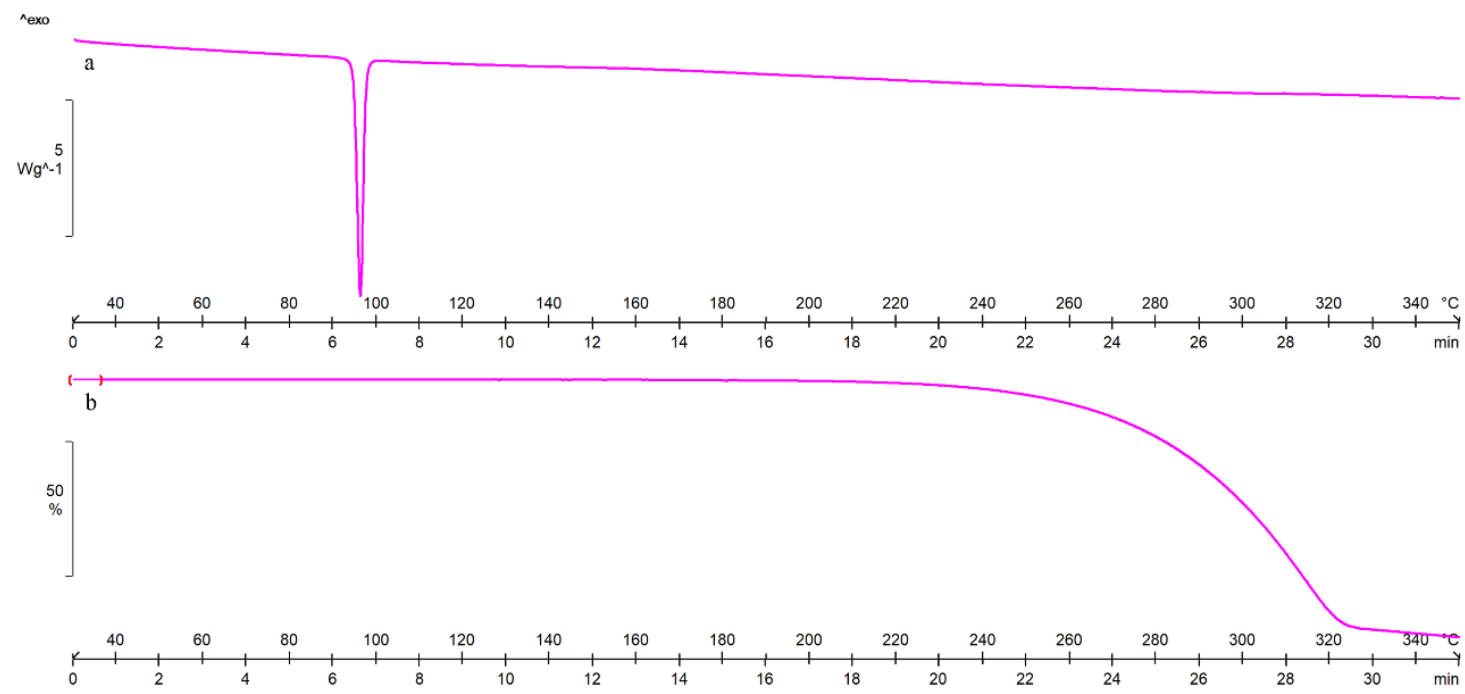

Figure 1. DSC (a) and TGA (b) profiles of the commercial PTB. 


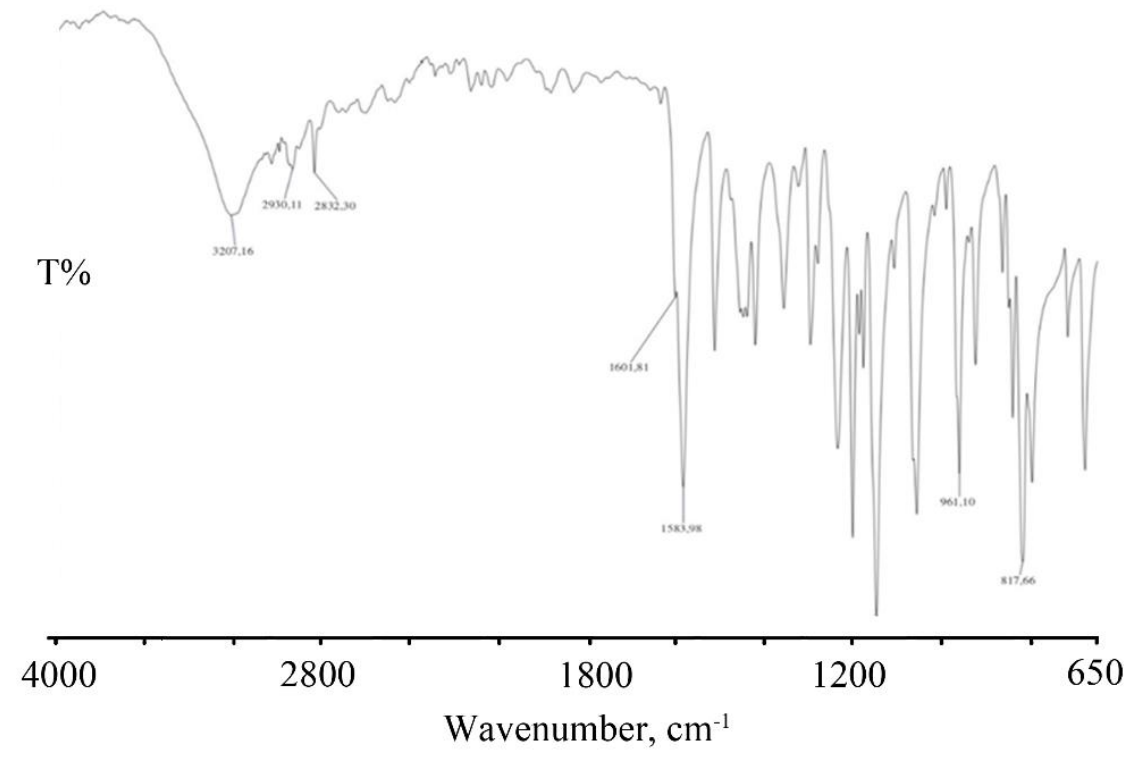

Figure 2. FT-IR spectrum of the commercial PTB.

To further characterize the solid state, PTB was analysed by FT-IR spectroscopy and in the spectrum obtained, the characteristic stretching and bending bands of the functional groups of the molecule are evident (Figure 2). In particular, in the spectrum are shown the broad band at $3207 \mathrm{~cm}^{-}$ ${ }^{1}$ due to the $\mathrm{OH}$ stretching vibration, the two bands at 2930 and $2832 \mathrm{~cm}^{-1}$ due to the stretching modes of the aromatic group, the band at $1601 \mathrm{~cm}^{-1}$ related to the stretching of the aromatic $\mathrm{C}-\mathrm{C}$ double bond and the band at $1583 \mathrm{~cm}^{-1}$ related to the stretching of olefinic $\mathrm{C}=\mathrm{C}$. The bands at 961 and $817 \mathrm{~cm}^{-1}$ are due to the vibrations of the $\mathrm{C}-\mathrm{H}$ groups and furthermore, the signal at $961 \mathrm{~cm}^{-1}$ is due to the stretching of the trans-olefinic $\mathrm{C}-\mathrm{H}$.

\subsection{Thermal and FT-IR characterization of the PTB-CDs binary systems}

In Figure 3 and 4 the DSC and TGA curves, respectively, of PTB, $\alpha-C D$, their physical mixture $(\mathrm{PM})$ and of the product obtained by kneading $(\mathrm{KN})$ are compared.

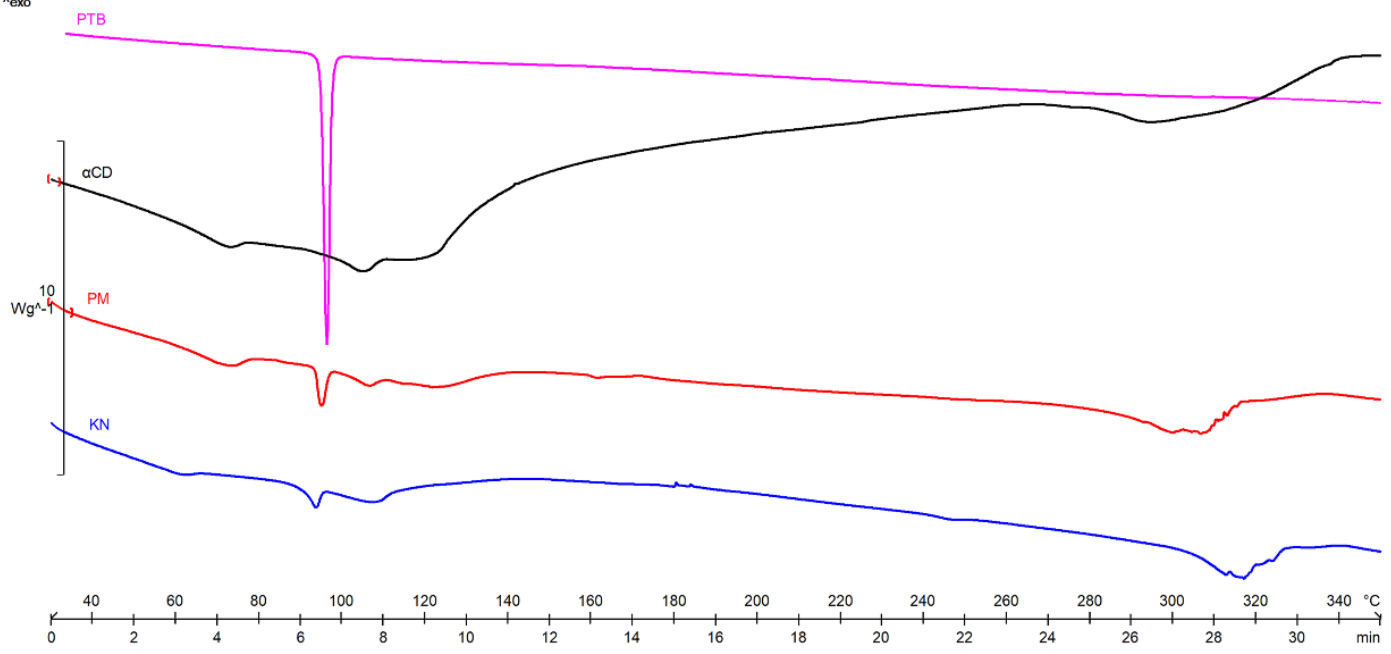

Figure 3. DSC profiles of the commercial PTB (violet), $\alpha-C D$ (black), PM (red) and KN (blue). 


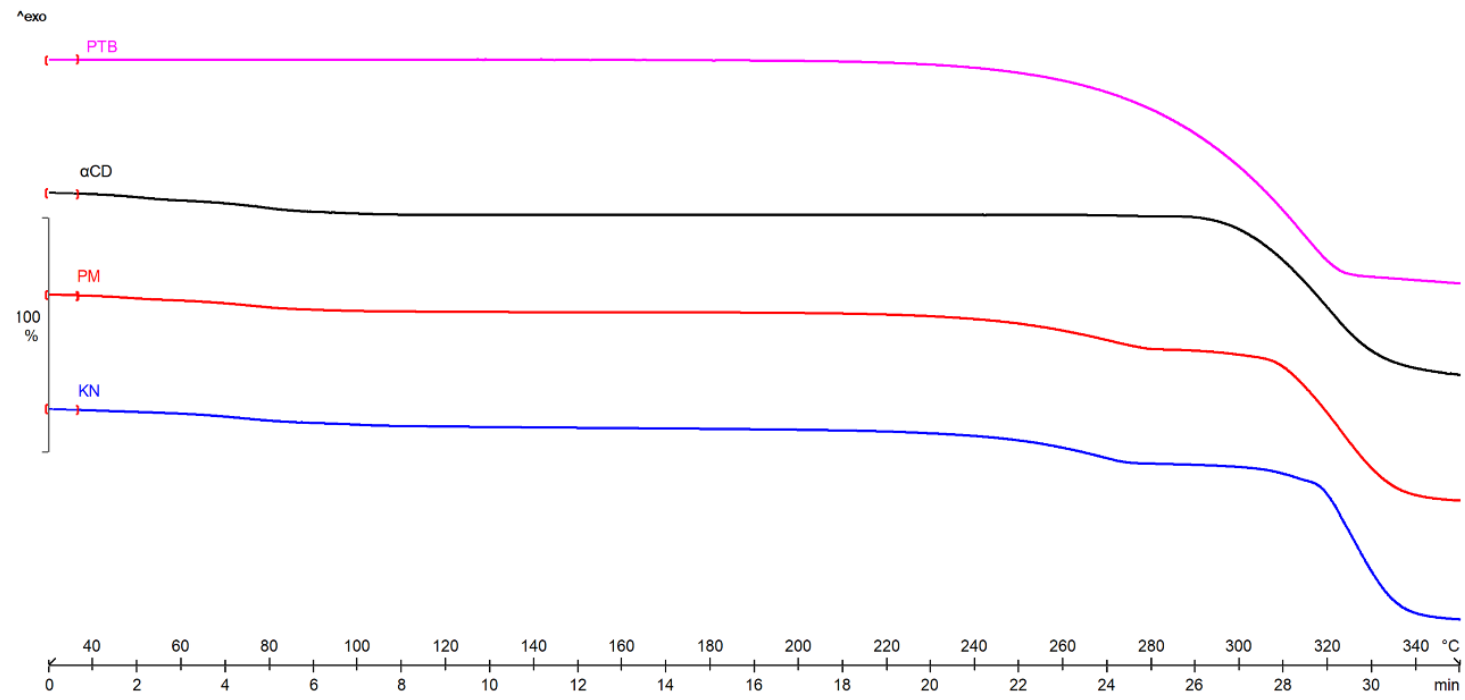

Figure 4. TGA profiles of the commercial PTB (violet), $\alpha-C D$ (black), PM (red) and KN (blue).

The DSC curve of $\alpha$-CD presents three distinct effects in the temperature range $30-150{ }^{\circ} \mathrm{C}$, attributed to sample dehydration with TGA total mass loss of $10.3 \pm 0.2 \%$ in agreement with the theoretical value $(10.0 \%)$. These effects are followed by melting with concomitant decomposition at around $270{ }^{\circ} \mathrm{C}$ as confirmed by TGA mass loss recorded in the same temperature range.

The drug melting endotherm is still evident both in the DSC curve of the PM and in the curve following the kneading treatment to which the binary mixture was submitted. However, the reduction of the melting enthalpy values by $17 \%$ and $42 \%$ for the $\mathrm{PM}$ and $\mathrm{KN}$ products respectively, relative to that of pure PTB is probably due to a partial complexation and/or amorphization of the system.

The DSC profiles of PTB, $\beta-C D$, their PM as such and after KN treatment are shown in Figure 5.

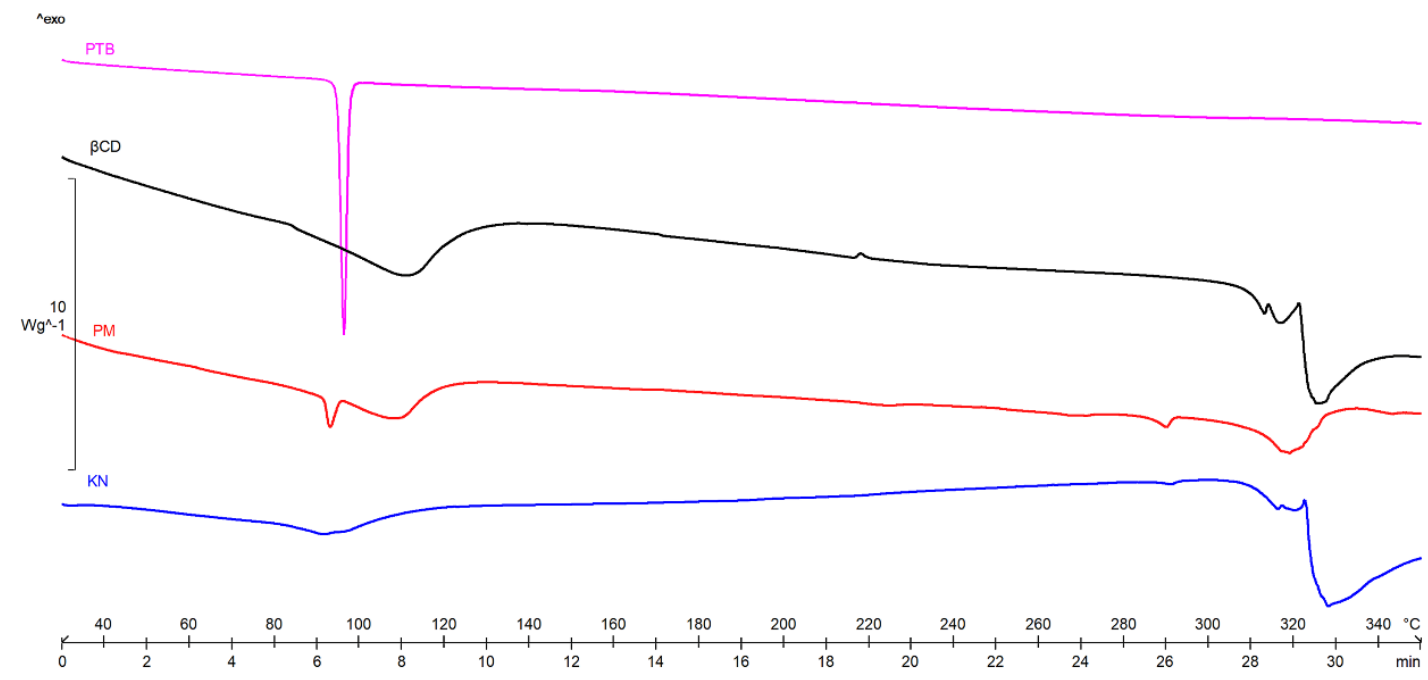

Figure 5. DSC profiles of the commercial PTB (violet), $\beta$-CD (black), their PM (red) and KN product (blue).

In the binary system with the higher homologue $\beta$-CD the drug melting peak is still evident in the PM but it disappeared completely in the treated system, indicating an inclusion of PTB in the hydrophobic CD cavity via the KN method. 
The thermal data are supported by FT-IR analysis (Figure 6). As the PM spectrum manifests as a combination of the bands of the two components, the KN product spectrum shows a shift or a disappearance of some typical bands of PTB (i.e. 1601 and $817 \mathrm{~cm}^{-1}$ ) confirming the formation of the complex.

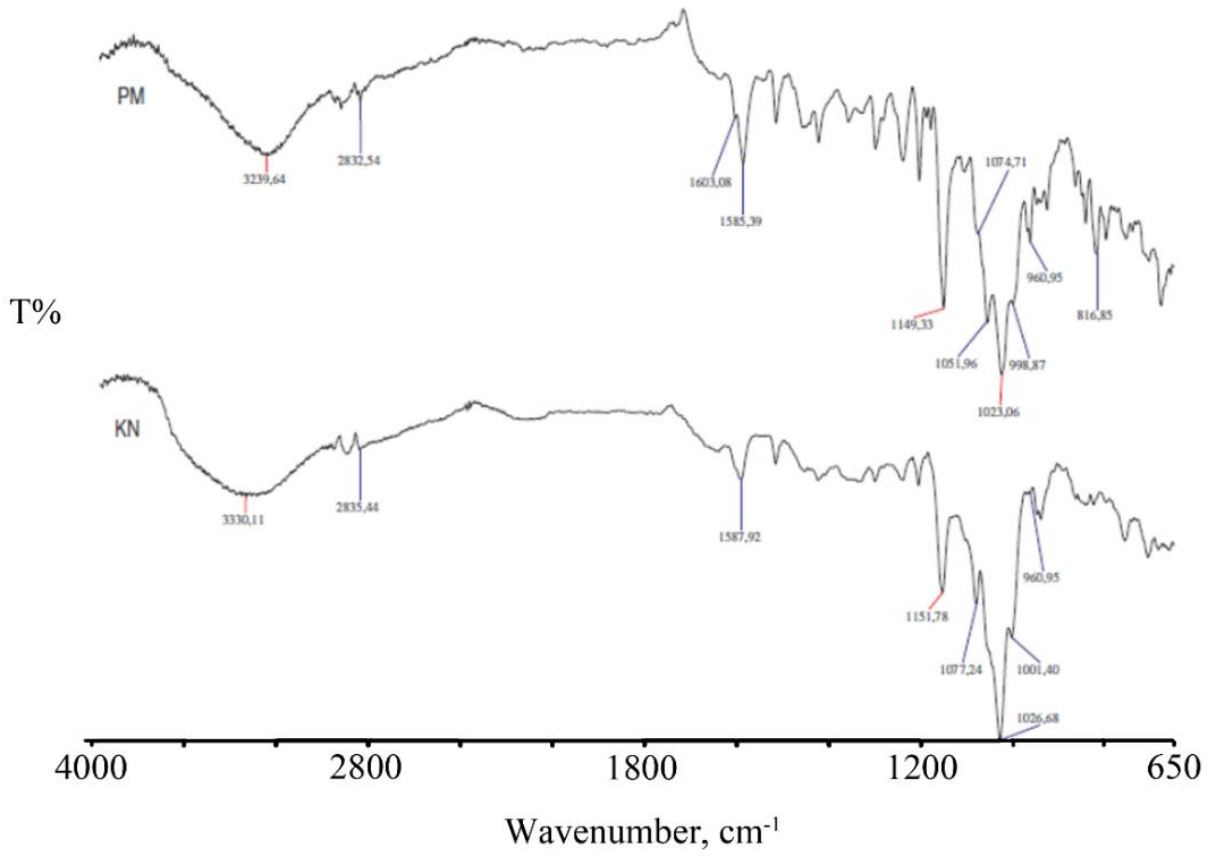

Figure 6. FT-IR spectra of PTB- $\beta$-CD PM and KN product.

The system with $\gamma$-CD presents thermal behaviours comparable to that shown earlier for the $\beta$ $\mathrm{CD}$ system. $\gamma$-CD is also able to complex PTB via $\mathrm{KN}$ treatment, as confirmed by FT-IR (data not shown).

\subsection{X-ray structural studies}

The isolation of single crystals of the complex of PTB with $\beta$-CD was pursued to determine the nature of the host-guest interactions using X-ray diffraction. The $\beta$-CD $P$ TB $10.2 \mathrm{H}_{2} \mathrm{O}$ complex crystallizes in the monoclinic space group $\mathrm{C} 2$. The asymmetric unit (ASU) in the crystal of the complex (Figure 7), comprises one molecule of the host (shown in a space-filling, cutaway view) and two components (A B) of guest disorder with site-occupancy $50 \%$ each (Water molecules have been omitted for clarity).

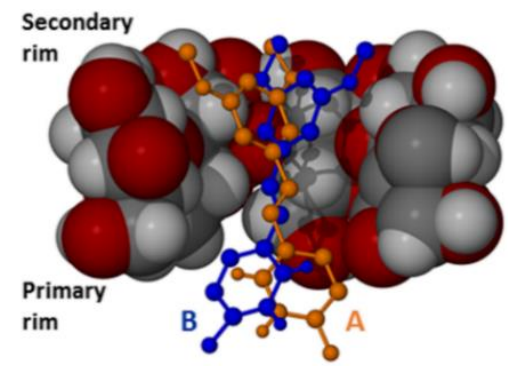

Figure 7. The ASU in the crystal of the complex $\beta$-CD.PTB· $10.2 \mathrm{H}_{2} \mathrm{O}$.

Applying the crystallographic $C_{2}$-axis rotation to the ASU generates the dimeric complex (Figure 8) which is maintained by $\mathrm{O}-\mathrm{H} \cdots \mathrm{O}$ hydrogen bonds at their interface (secondary---secondary rims). 

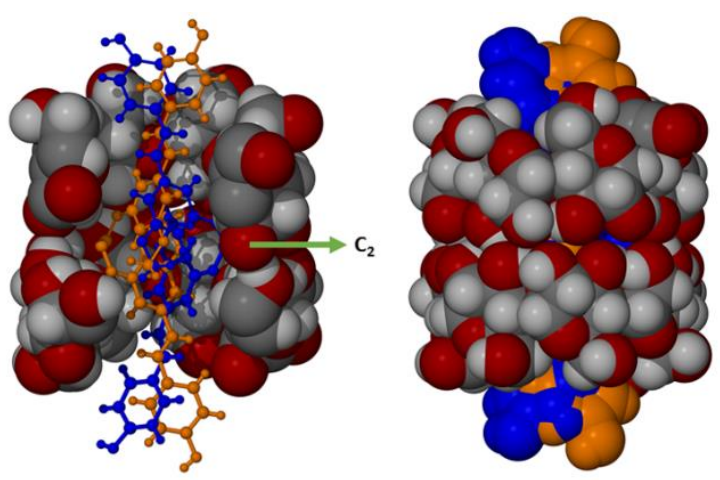

Figure 8. The dimeric complex shown in cutaway view (left), and with all atoms included and drawn in space-filling mode (right).

A microcrystalline product obtained by kneading $\gamma$-CD and PTB was investigated by powder Xray diffraction. The close resemblance of the PXRD pattern of this phase to that of a known isostructural $\gamma$-CD complex showed unequivocally that it is an inclusion complex crystallizing in the tetragonal space group P4212.

A solution of crystalline $\gamma$-CD.PTB complex obtained by co-precipitation was also characterized by ${ }^{1} \mathrm{H}-\mathrm{NMR}$ spectroscopy revealing a 1:1 host-guest composition (data not shown).

\section{Discussion}

The present investigation focused on the feasibility of including the antioxidant PTB in the native cyclodextrins $\alpha-, \beta-$, and $\gamma-C D$, the results indicating little or no evidence of the affinity of PTB to complex with $\alpha-C D$ using the kneading method. However, with the higher homologues $\beta$-CD and $\gamma$ $\mathrm{CD}$ a definitive indication of complexation was first evident from thermal analysis and subsequently corroborated by FT-IR and ${ }^{1} \mathrm{H}-\mathrm{NMR}$ spectroscopy, as well as PXRD and single-crystal X-ray diffraction methods.

Author Contributions: Conceptualization, L.C., M.S. and M.R.C.; methodology, L.C., M.S. and A.I.V: validation, M.S., L.C., A.I.V. and M.R.C.; resources, M.S. and M.R.C.; data curation, M.S., L.C., A.I.V. and M.R.C.; writingoriginal draft preparation, L.C. and M.R.C.; writing - review and editing, L.C.; M.S.; M.C.B.; A.I.V. and M.R.C.; supervision, M.S., L.C., and M.R.C.; project administration, M.S. and M.R.C.

Acknowledgments: M.R.C. thanks the University of Cape Town for research support.

Conflicts of Interest: The authors declare no conflict of interest.

\section{References}

1. McCormack, D.; McFadden, D. A review of pterostilbene antioxidant activity and disease modification. Oxid Med Cell Longev. 2013, 575482, doi: 10.1155/2013/575482.

2. Zhiqiang, M.; Xiaoyan, Z.; Liqun, X.; Dong, L.; Shouyin, D.; Weimiao, L.; Jiao, Z.; Hongmei, Z.; Xiaofei, L.; Jing, H.; Xiaolong, Y. Pterostilbene: Mechanisms of its action as oncostatic agent in cell models and in vivo studies. Pharmacol. Res. 2019, 145, 104265, doi.org/10.1016/j.phrs.2019.104265.

3. Pinho, E.; Grootveld, M.; Soares, G.; Henriques, M. Cyclodextrins as encapsulation agents for plant bioactive compounds. Carbohydr. Polym. 2014, 101, 21-135, doi.org/10.1016/j.carbpol.2013.08.078.

4. Jacob, S.; Nair, A.B. Cyclodextrin complexes: Perspective from drug delivery and formulation. Drug Dev. Res. 2018, 79, 201-217, doi.org/10.1002/ddr.21452.

(C) 2020 by the authors; licensee MDPI, Basel, Switzerland. This article is an open access article distributed under the terms and conditions of the Creative Commons by Attribution (CC-BY) license (http://creativecommons.org/licenses/by/4.0/). 